\title{
Flexible rotation of transverse optical field for 2D self-accelerating beams with a designated trajectory
}

\author{
Lei Zhu' ${ }^{1}$, Xuesong Zhao ${ }^{1}$, Chen $\mathrm{Liu}^{1}$, Songnian $\mathrm{Fu}^{2 *}$, Yuncai Wang ${ }^{2}$ and \\ Yuwen Qin ${ }^{2}$
}

Self-accelerating beams have the unusual ability to remain diffraction-free while undergo the transverse shift during the free-space propagation. We theoretically identify that the transverse optical field distribution of 2D self-accelerating beam is determined by the selection of the transverse Cartesian coordinates, when the caustic method is utilized for its trajectory design. Based on the coordinate-rotation method, we experimentally demonstrate a scheme to flexibly manipulate the rotation of transverse optical field for 2D self-accelerating beams under the condition of a designated trajectory. With this scheme, the transverse optical field can be rotated within a range of 90 degrees, especially when the trajectory of $2 \mathrm{D}$ self-accelerating beams needs to be maintained for free-space photonic interconnection.

Keywords: self-accelerating beams; optical field; caustic

Zhu L, Zhao XS, Liu C, Fu SN, Wang YC et al. Flexible rotation of transverse optical field for 2D self-accelerating beams with a designated trajectory. Opto-Electron Adv 4, 200021 (2021).

\section{Introduction}

Self-accelerating beams are the solutions to the wave equation, with intensity peak traveling along a curved trajectory during the free-space propagation ${ }^{1}$. The concept of snake beams has been proposed by Rosen and Yariv in 1995'. However, it was until 2007 that Siviloglou and Christodoulides first experimentally generated the Airy beam with a parabolic trajectory ${ }^{3,4}$. Self-accelerating beams have attracted worldwide attentions in the field of high-resolution imaging ${ }^{5}$, particle manipulation ${ }^{6}$, light bullets $^{7,8}$, micro-machining ${ }^{9}$, and obstacle evasion optical communication ${ }^{10,11}$. Self-accelerating beams also have broad and important applications beyond optics, due to the similar form of wave equations, so that self-accelerating beams can be easily introduced into acoustic waves $^{12}$, electronic waves ${ }^{13}$, and plasmonic waves ${ }^{14-16}$.
Generally, the main lobe of self-accelerating beams possesses most energy of transverse optical field and propagates along a curved trajectory. Consequently, previous research works and applications were mainly focused on the main lobe of self-accelerating beams. For practical applications, self-accelerating beams with flexible trajectory manipulation are required. One way is to find other analytical solutions to the wave equation, such as Bessel-like self-accelerating beam with circle trajectory $^{17}$, Mathieu beam with elliptical trajectory ${ }^{18}$, and Weber beam with parabolic trajectory ${ }^{19}$. However, an efficient strategy to generate the accelerating beam with an arbitrary trajectory is to use the optical caustic strategy ${ }^{20,21}$, even though the generated beam is not the exact accelerating non-diffraction solution to the wave equation. The caustic method was first proposed by Greenfield in $2011^{22}$, then it has been extended from

1Wuhan National Laboratory for Optoelectronics, and School of Optical and Electronic Information, Huazhong University of Science and Technology, Wuhan 430074, China; ${ }^{2}$ School of Information Engineering, Guangdong University of Technology, and Guangdong Provincial Key Laboratory of Photonics Information Technology, Guangzhou 510006, China.

*Correspondence: SN Fu, E-mail: songnian@gdut.edu.cn

Received: 22 June 2020; Accepted: 31 July 2020; Published: 25 March 2021 
paraxial beams to non-paraxial beams ${ }^{18,19}$ in order to obtain large bending angle, and from the convex trajectory to non-convex trajectory ${ }^{23,24}$. However, unlike Gaussian beam or Bessel beam, since the light rays have to be distributed outside the local convex part of the trajectory according to the optical caustic, the transverse optical field of 2D self-accelerating beams with a curved trajectory is definitely not circularly symmetrical around the main lobe. For example, the transverse optical field of $2 \mathrm{D}$ Airy beam is mainly located at a quarter of the transverse plane. Such asymmetrical distribution of side lobes has to be taken into account, when $2 \mathrm{D}$ self-accelerating beams are involved for some optical field distribution sensitive applications, such as micro-machining, particle manipulation, and free-space photonic interconnection. Although the transverse rotation of optical field for 2D self-accelerating beams can be realized by loading a rotated phase pattern into the spatial light modulator (SLM), the corresponding trajectory varies as well. Such a solution may not be ideal for some practical applications, because the main lobe possesses the most energy of $2 \mathrm{D}$ self-accelerating beams, and the trajectory of main lobe has to be maintained as well. Therefore, flexible manipulation of transverse optical field without changing the designated trajectory is highly desired.

In this submission, we firstly identify that the transverse optical field is closely associated with the selection of transverse Cartesian coordinates, when 2D self-accelerating beams are generated by the optical caustic method. Consequently, we propose a coordinate-rotation based strategy, in order to realize optical field rotation within 90 degrees. Finally, a proof-of-concept experiment to verify our proposed method is demonstrated for the application of obstacle evasion free-space photonics interconnection.

\section{Operation principle of manipulating transverse field distribution}

Generally, the free-space propagation of optical field can be expressed as the integral of angular spectrum diffraction

$$
\begin{aligned}
& E(X, Z) \\
& =\frac{1}{2 \pi} \int A\left(k_{x}\right) \exp \left\{\mathrm{i}\left[k_{x} X+\sqrt{k^{2}-k_{x}^{2}} Z+\phi\left(k_{x}\right)\right]\right\} \mathrm{d} k_{x} \\
& =\frac{1}{2 \pi} \int A\left(k_{x}\right) \exp \left[\mathrm{i} \psi\left(k_{x}\right)\right] \mathrm{d} k_{x},
\end{aligned}
$$

where $A\left(k_{x}\right)$ and $\phi\left(k_{x}\right)$ are the amplitude and phase distribution of the initial angular spectrum, $k$ denotes the wavenumber, and $k_{x}$ is the wavenumber in $X$ direction. Applying the stationary-phase approximation ${ }^{22,25}$ to the integral, the main contribution to $E(X, Z)$ comes from the stationary points where

$$
\frac{\mathrm{d} \psi\left(k_{x}\right)}{\mathrm{d} k_{x}}=X-\frac{k_{x}}{\sqrt{k^{2}-k_{x}^{2}}} Z+\varphi^{\prime}\left(k_{x}\right)=0 .
$$

For a specific spatial frequency $k_{x}$, Eq. (2) indicates that the stationary points are along a straight line. As a result, the propagation behavior of optical field can be simplified by a series of light rays, which can be analyzed by geometric optics. Besides, from the view of real space $^{22}$, applying the stationary-phase approximation to the Fresnel diffraction integral, we can obtain the same conclusion.

The bending trajectory of self-accelerating beams can be explained by the caustic phenomenon, where the light rays are tangent to the bending trajectory, instead of being focused to a specific point. Consequently, in order to realize a self-accelerating beam with an arbitrary trajectory, we can first get a family of light rays which are tangent to the desired trajectory, then back-trace them to obtain the initial optical field or its spatial spectrum. Since the light rays are tangent to the trajectory, they have to be distributed at the convex side of the trajectory. Meanwhile, 2D self-accelerating beam can be regarded as the combination of two $1 \mathrm{D}$ self-accelerating beams in planes of $(X, 0, Z)$ and $(0, Y, Z)$. As shown in Fig. 1, 2D self-accelerating beam with a convex trajectory is taken as an example. The projection of the curved trajectory on the initial plane is highlighted as a red curve in Figs. 1(c) and $1(\mathrm{f})$. With the trajectory decomposed into planes of $(X, 0, Z)$ and $(0, Y, Z)$, the $X$ and $Y$ components of trajectory and light rays are shown as red curves and blue light rays in Figs. 1(a) and 1(b), respectively. As we can see, the light rays are located at the positive direction of $X$ axis and the negative position of $Y$ direction. Hence, the corresponding optical field of point $A$ in the default Cartesian coordinates is shown as the blue area of Fig. 1(c). However, under the condition of the fixed freespace trajectory, it can be decomposed to different coordinate pairs. Based on the new coordinates which are rotated counterclockwise with an angle of $\theta$ from the initial one, the components of the trajectory and light rays vary, as shown in Figs. 1(d) and 1(e). Accordingly, the optical field distribution is coordinate-dependent and can be rotated with an angle of $\theta$, as shown in Fig. 1(f). Therefore, we can conclude that the transverse optical 
field is associated with the selection of the transverse Cartesian coordinates, when $2 \mathrm{D}$ self-accelerating beams are generated by composing two independent $1 \mathrm{D}$ self-accelerating beams.

\section{Simulation results and experimental verifications}

In order to verify the coordinate-dependent optical field distribution, an experimental characterization setup is schematically shown in Fig. 2. At the output of standard single mode fiber, the light beam from a distributed feedback (DFB) laser at operation wavelength of $1550 \mathrm{~nm}$ is collimated to a Gaussian beam with a waist radius of about $1.75 \mathrm{~mm}$. After passing through a linear polarizer
(P) and a beam splitter (BS), the beam is spatially modulated by a reflective SLM (PLUTO-TEL-013, HOLOEYE) with a resolution of $1920 \times 1080$ and a pixel pitch of $8 \mu \mathrm{m}$. Fourier modulation is utilized in order to generate highquality $2 \mathrm{D}$ self-accelerating beams, in comparison with the real-space modulation. Therefore, a lens with a focal length of $0.2 \mathrm{~m}$ is set after the SLM, and the $2 \mathrm{D}$ self-accelerating beams can be obtained behind the lens.

\section{Phase pattern}

Generally, there are two approaches to generate self-accelerating beams, including Fourier space modulation with the help of a lens and real space modulation. Our experimental setup is based on the spatial spectrum
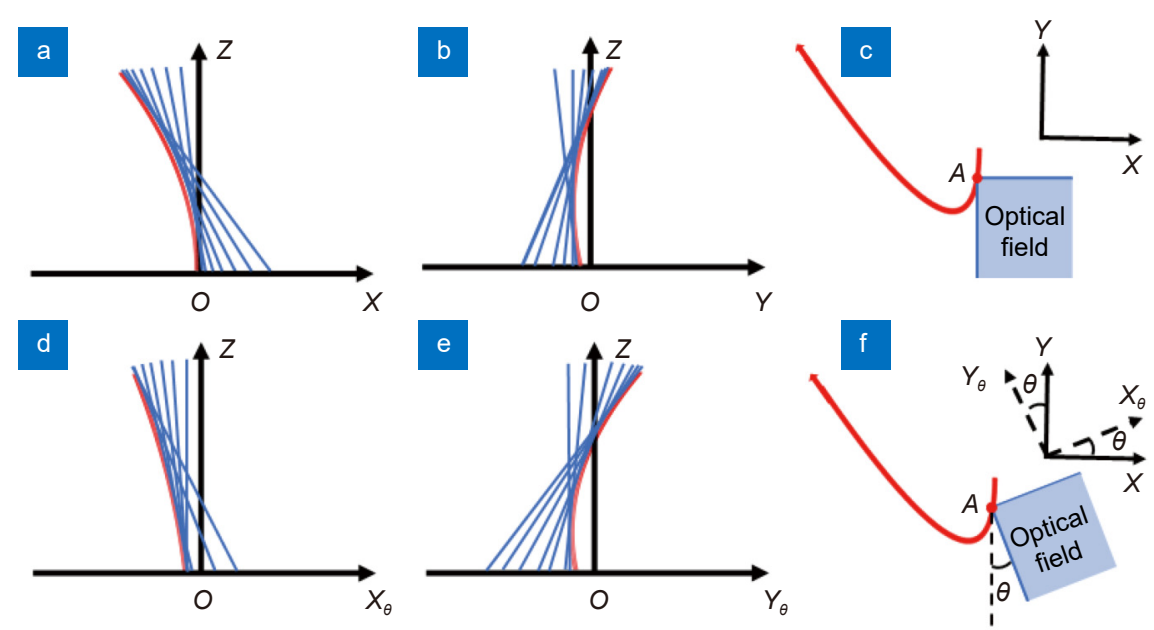

Fig. 1 | Generation of 2D self-accelerating beam based on optical caustic and the rotation principle of transverse optical field for 2D self-accelerating beam. Two perpendicular components (a)-(b) of trajectory and light rays in default Cartesian coordinates. (c) The projection of multiplexed trajectory and the distribution of transverse optical field in default Cartesian coordinates; two perpendicular components (d)-(e) of trajectory and light rays in rotated Cartesian coordinates. (f) The projection of multiplexed trajectory and the rotated distribution of transverse optical field in rotated Cartesian coordinates.

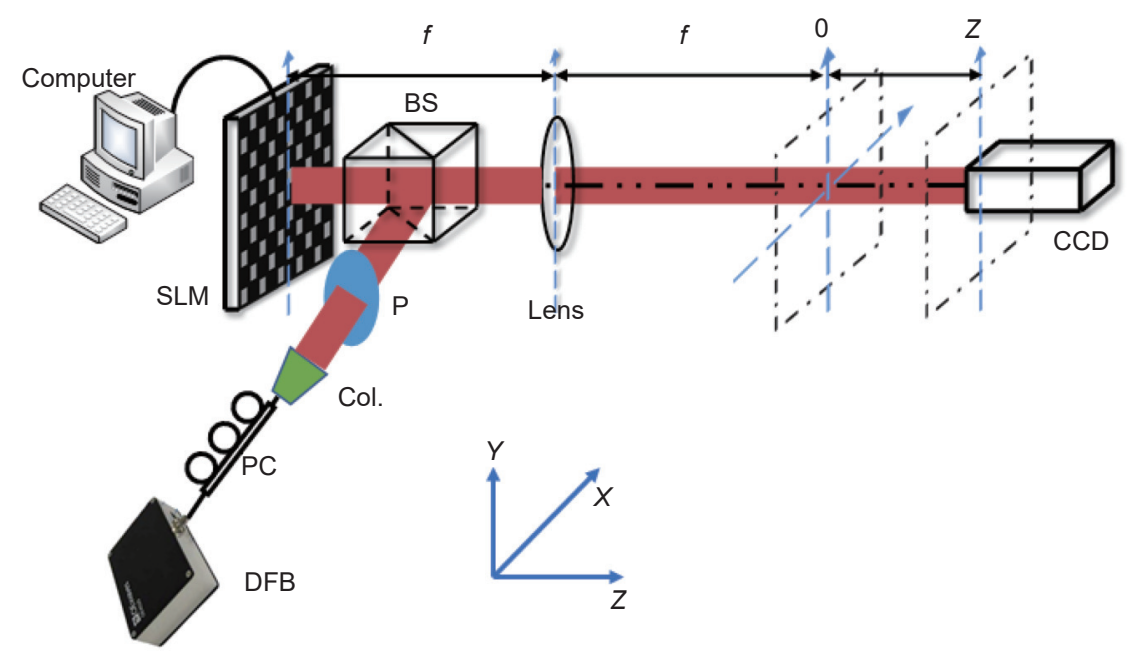

Fig. 2 | Experimental setup for the optical field rotation of 2D self-accelerating beams. DFB: distributed feedback laser; Col.: collimator; PC: polarizer controller; P: polarizer; BS: beam splitter; SLM: spatial light modulator. 
modulation in Fourier space. Both two approaches can be unified in the phase space ${ }^{26}$ by the Wigner distribution function (WDF) ${ }^{27}$. The physical meaning of WDF in optics can be approximately interpreted as the intensity of a light ray with a specific position and direction. We discuss the general curved trajectory with the form of $X=f(Z), Y=g(Z)$. After two orthogonal coordinates are rotated anticlockwise with an angle of $\theta$, the designated trajectory under the new coordinates is

$$
\left[\begin{array}{c}
X_{\theta} \\
Y_{\theta}
\end{array}\right]=\left[\begin{array}{cc}
\cos \theta & \sin \theta \\
-\sin \theta & \cos \theta
\end{array}\right]\left[\begin{array}{l}
f(Z) \\
g(Z)
\end{array}\right] .
$$

Since the light rays are tangent to the designated trajectory, after the coordinate rotation, emitting positions of light rays from the initial plane (plane $Z=0$ ) can be obtained as

$$
\left[\begin{array}{l}
x_{0, \theta} \\
y_{0, \theta}
\end{array}\right]=\left[\begin{array}{cc}
\cos \theta & \sin \theta \\
-\sin \theta & \cos \theta
\end{array}\right]\left[\begin{array}{c}
f(Z)-Z f^{\prime}(Z) \\
g(Z)-Z g^{\prime}(Z)
\end{array}\right],
$$

and the direction of each light ray is

$$
\left[\begin{array}{l}
k_{0, x, \theta} \\
k_{0, y, \theta}
\end{array}\right]=k\left[\begin{array}{cc}
\cos \theta & \sin \theta \\
-\sin \theta & \cos \theta
\end{array}\right]\left[\begin{array}{l}
f^{\prime}(Z) \\
g^{\prime}(Z)
\end{array}\right] .
$$

Next, according the reference ${ }^{26}$, the WDF of initial optical field can be expressed as

$$
\begin{aligned}
& W_{\theta}\left(x_{\theta}, y_{\theta}, k_{x, \theta}, k_{y, \theta}\right) \\
& =\int \delta\left[x_{\theta}-x_{0, \theta}, y_{\theta}-y_{0, \theta}\right] \delta\left[k_{x, \theta}-k_{0, x, \theta}, k_{y, \theta}-k_{0, y, \theta}\right] \mathrm{d} Z,
\end{aligned}
$$

where $x_{\theta}$ and $y_{\theta}$ denote the coordinate variables of the initial plane $\left(X_{\theta}, Y_{\theta}, 0\right)$. The phase pattern to be loaded on the SLM is determined by the phase distribution of the calculated optical field at the initial plane, which can be obtained according to the properties of $\mathrm{WDF}^{26}$,

$$
\phi_{\theta}=\int-\frac{\int x_{\theta} y_{\theta} W_{\theta} \mathrm{d} x_{\theta} \mathrm{d} y_{\theta}}{\int W_{\theta} \mathrm{d} x_{\theta} \mathrm{d} y_{\theta}} \mathrm{d} k_{x, \theta} \mathrm{d} k_{y, \theta},
$$

where, for a lens-based Fourier transformation system, $k_{x, \theta}=2 \pi x_{\theta} /(\lambda f), k_{y, \theta}=2 \pi y_{\theta} /(\lambda f), f$ is the focal length oflens. Duetotherelationshipsin the right-hand coordinate system including $x_{\theta+90^{\circ}}=y_{\theta}, y_{\theta+90^{\circ}}=-x_{\theta}, x_{0, \theta+90^{\circ}}=$ $y_{0, \theta}$ and $y_{0, \theta+90^{\circ}}=-x_{0, \theta}$, we can derive

$$
\begin{aligned}
& W_{\theta+90^{\circ}}=\int \delta\left(x_{\theta+90^{\circ}}-x_{0, \theta+90^{\circ}}, y_{\theta+90^{\circ}}-y_{0, \theta+90^{\circ}}\right) \\
& \times \delta\left(k_{x, \theta+90^{\circ}}-k_{0, x, \theta+90^{\circ}}, k_{y, \theta+90^{\circ}}-k_{0, y, \theta+90^{\circ}}\right) \mathrm{d} Z \\
& =\int \delta\left(y_{\theta}-y_{0, \theta},-x_{\theta}+x_{0, \theta}\right) \\
& \times \delta\left(k_{y, \theta}-k_{0, y, \theta},-k_{x, \theta}+k_{0, x, \theta}\right) \mathrm{d} Z=W_{\theta} .
\end{aligned}
$$

It indicates that the Wigner distribution function for our scheme varies with a period of $90^{\circ}$. Then, we can further obtain $\phi_{\theta+90^{\circ}}=\phi_{\theta}$. Therefore, the phase patterns for the transverse field with rotation angles of $\theta+90^{\circ}$ and $\theta$ are identical. As a result, the transverse optical field can rotate within $90^{\circ}$ while the trajectory is maintained without the modification. Since the light rays have to be located at the convex part of the trajectory, the range of rotation angle cannot exceed $90^{\circ}$ with our proposed method.

\section{Simulation results}

As a typical self-accelerating beam with the convex trajectory, 2D Airy beam ${ }^{4}$ is selected to verify the proposed optical field rotation technique. We set the trajectory of 2D Airy beam as

$$
\left\{\begin{array}{rl}
X= & 7.7896 \times 10^{-3} Z^{2}-3.9470 \times 10^{-3} Z \\
& +0.8750 \times 10^{-3} / \mathrm{m} \\
Y= & 5.5445 \times 10^{-3} Z^{2}-5.2862 \times 10^{-3} Z \\
& +1.6800 \times 10^{-3} / \mathrm{m}
\end{array} .\right.
$$

The original phase pattern in the default Cartesian coordinates, is shown as Fig. 3(a). The corresponding 3D optical field distribution is calculated in Fig. 3(b), and the red curve denotes the trajectory. The projection of trajectory is shown in Fig. 3(c), which is consistent with Eq. (9). From Fig. 3(b), we can find that the boundary lines of transverse optical field are parallel to the $X$ and $Y$ axis, and the optical field is mainly distributed within this right-angle area. If the rotation of optical distribution is desired, a straightforward way is to simply rotate the phase pattern in the default Cartesian coordinates around its center with the specific angle, like in Fig. 3(d) with phase pattern rotation angle of $-30^{\circ}$. The calculated 3D optical distribution of beam generated by the rotated phase pattern is shown as Fig. 3(e). The optical field distribution is rotated with an angle of $-30^{\circ}$. However, the trajectory is rotated around $Z$ axis as well, as shown in Fig. 3(f), which is not preferred. On the contrary, with the re-calculated phase patterns by our proposed method, as shown in Figs. 3(g) and 3(j), the optical distribution is rotated with an angle of $-30^{\circ}$ and $15^{\circ}$, and the trajectory can be maintained without the modification, as shown in Figs. 3(h), 3(i), 3(k) and 3(l).

\section{Experimental results}

The phase patterns to be loaded on the SLM for generating the $2 \mathrm{D}$ Airy beams with rotation angles of $-30^{\circ}, 0^{\circ}$ and $15^{\circ}$ are calculated, respectively, according to Eq. (6) 
and (7), as shown in Figs. 4(a-c). The white dotted lines in Figs. $4(\mathrm{~d}-\mathrm{u})$ are the projection of the designated trajectory on the initial plane. The intensity profiles are captured every $5 \mathrm{~cm}$ from the back focal plane of lens, but only the results at distance of $0,0.3 \mathrm{~m}$ and $0.6 \mathrm{~m}$ are presented here. In Fig. 4, we find the experimental results are in good agreement with our simulation results. The main lobes of the experimentally generated light beams at distance of $0.3 \mathrm{~m}$ are precisely located at the white dotted projection of trajectory, but there occur few deviations at distance of 0 and $0.6 \mathrm{~m}$. We infer that the optical path is not fully aligned. However, we can con- clude that the transverse optical field of 2D Airy beams generated in our experiments can be successfully rotated in a manageable way.

\section{Application of free-space photonic interconnection}

When an obstacle occurs at the light path of free-space photonic interconnection system, the interruption happens. Therefore, a curved trajectory of $2 \mathrm{D}$ self-accelerating beams is helpful to realize the obstacle evasion photonic interconnection, because the appropriate trajectory of $2 \mathrm{D}$ self-accelerating beams can be designed to
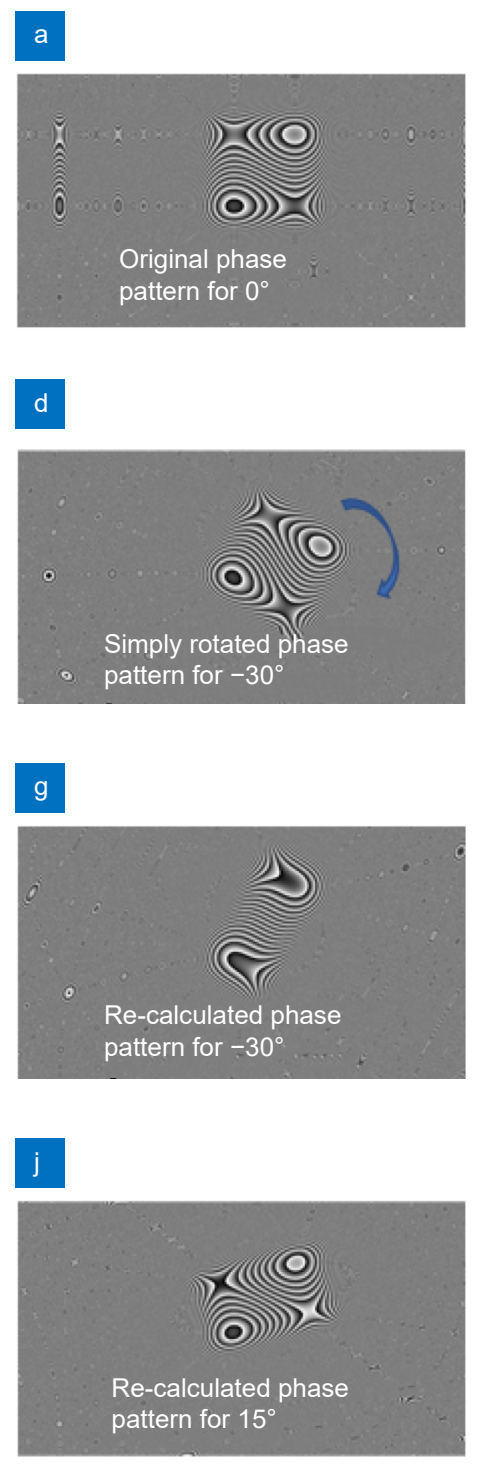
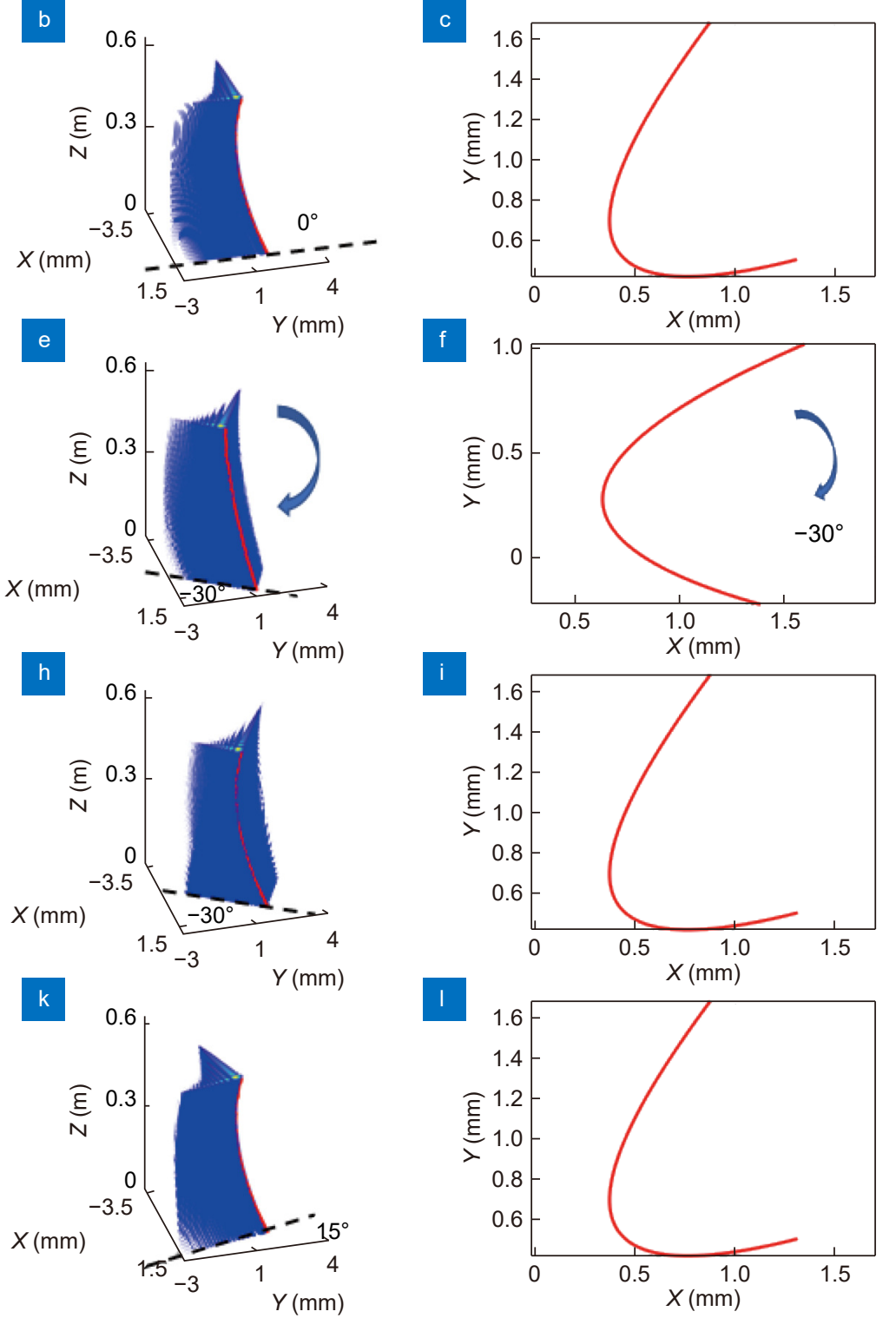

Fig. 3 | Different phase patterns and their calculated 3D optical distribution and trajectories. (a) Original phase pattern and its (b) 3D optical distribution and (c) the projection of trajectory. (d) Simply rotated phase pattern for $-30^{\circ}$ and corresponding (e) 3D optical distribution and (f) the projection of trajectory. (g), (j) Re-calculated phase patterns for $-30^{\circ}$ and $15^{\circ}$, and (h), (k) their $3 \mathrm{D}$ optical distribution and (i), (I) the projection of trajectory. 
$\theta=-30^{\circ}$
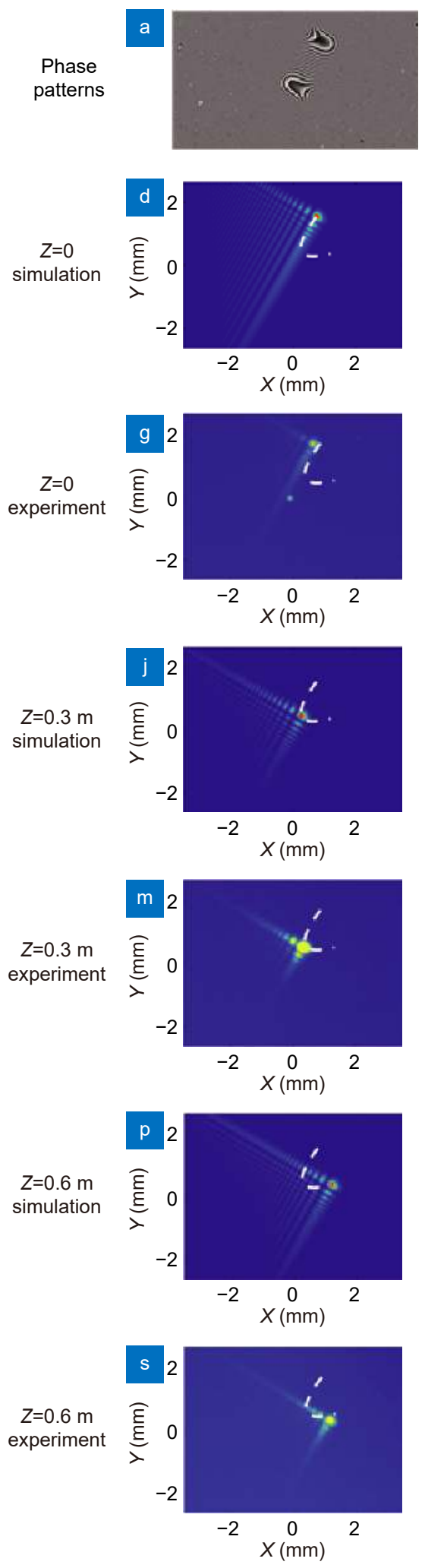

$\theta=0^{\circ}$

b
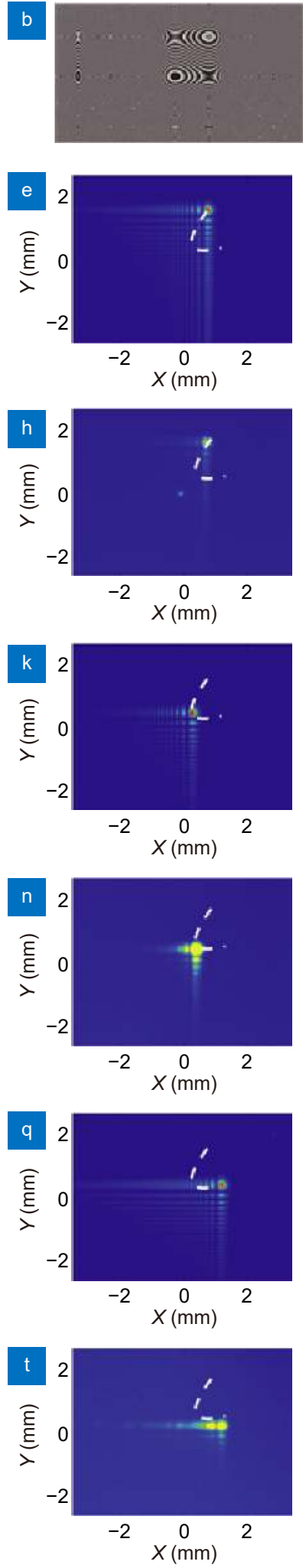

$\theta=15^{\circ}$
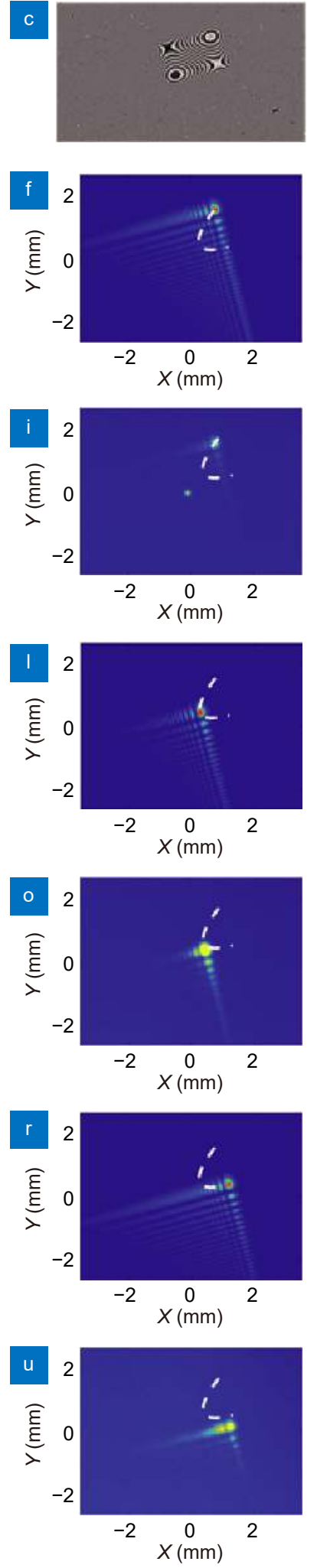

Fig. 4 | Calculated and experimental results for the optical field rotation of 2D Airy beam at different propagation distances. (a - c) Calculated phase patterns. $(\mathbf{d}-\mathbf{f}),(\mathbf{j}-\mathbf{I}),(\mathbf{p}-\mathbf{r})$ Simulated and $(\mathbf{g}-\mathbf{i}),(\mathbf{m}-\mathbf{o}),(\mathbf{s}-\mathbf{u})$ experimental intensity profiles at distance of $0,0.3 \mathrm{~m}$ and $0.6 \mathrm{~m}$ with a rotation angle of $-30^{\circ}, 0^{\circ}$ and $15^{\circ}$, respectively. 
evade the obstacle. We assume that the positions of transmitter and receiver are at $T(0,0,0)$ and $R(0,0,0.6 \mathrm{~m})$, respectively. A thin opaque circle obstacle with its quarter removed, whose center is put at the position of $C(1 \mathrm{~mm}, 1 \mathrm{~mm}, 0.3 \mathrm{~m})$, is set at the distance of $0.3 \mathrm{~m}$, as shown in Fig. 5(a). Point $O$ denotes the intersection of $Z$ axis and obstacle plane. The obstacle can be rotated around its center point $C$ freely along the vertical dotted line with an angle of $\beta$. To evade the obstacle, the trajectory of $2 \mathrm{D}$ Airy beam must pass through points $T, C$ and $R$ successively. The trajectory of $2 \mathrm{D}$ Airy beam is set as $X=Y=-11.4305 \times 10^{-3} Z^{2}+6.8583 \times 10^{-3} Z / m$, as shown in the red parabolic curve of Fig. 5(a). Under such a trajectory, the main lobe of $2 \mathrm{D}$ Airy beam is able to bypass the obstacle and be successfully collected by the receiver. If the optical field of the generated beam is fixed, the bypassed energy is different when the obstacle angles $\beta$ are varied. Therefore, the bypassed energy can be optimized by the selection of 2D Airy beam with an appropriate optical field distribution. The 2D Airy beam with the rotated optical field is generated with an angle of $\theta$ from $-45^{\circ}$ to $45^{\circ}$. Meanwhile, the obstacle is experimentally rotated with $\beta=-15^{\circ}, 0^{\circ}$ and $30^{\circ}$, respectively. After the obstacle, another lens is used to converge the bypassed light to a small spot and an integral sphere is used to col- lect the bypassed optical energy. The calculated results together with the experimental measurements after the normalization are shown in Figs. $5(b-d)$. Due to the non-fully aligned optical axis and mechanical variation, it is challenging to set the main lobe of Airy beam at $z=0.3 \mathrm{~m}$ locate at point $C$ precisely when the obstacle is rotated. The position deviation between main lobe and point $C$ leads to some transmittance differences between the simulation and experimental results. However, it is clearly observed that, when the rotation angles of obstacle and the optical field are identical, the bypassed optical power can approach the maximum value. Consequently, after we design an appropriate trajectory to evade obstacle, the interconnection performance can be further optimized when the obstacle structure and the corresponding optical field distribution are simultaneously taken into consideration.

\section{Conclusions}

We have demonstrated a scheme to realize a flexible rotation of transverse field for $2 \mathrm{D}$ self-accelerating beams under the condition of a fixed trajectory. Based on the rotation of transverse Cartesian coordinates, the transverse optical field can be flexibly rotated within 90 degrees. By selecting 2D Airy beam as a simple 2D
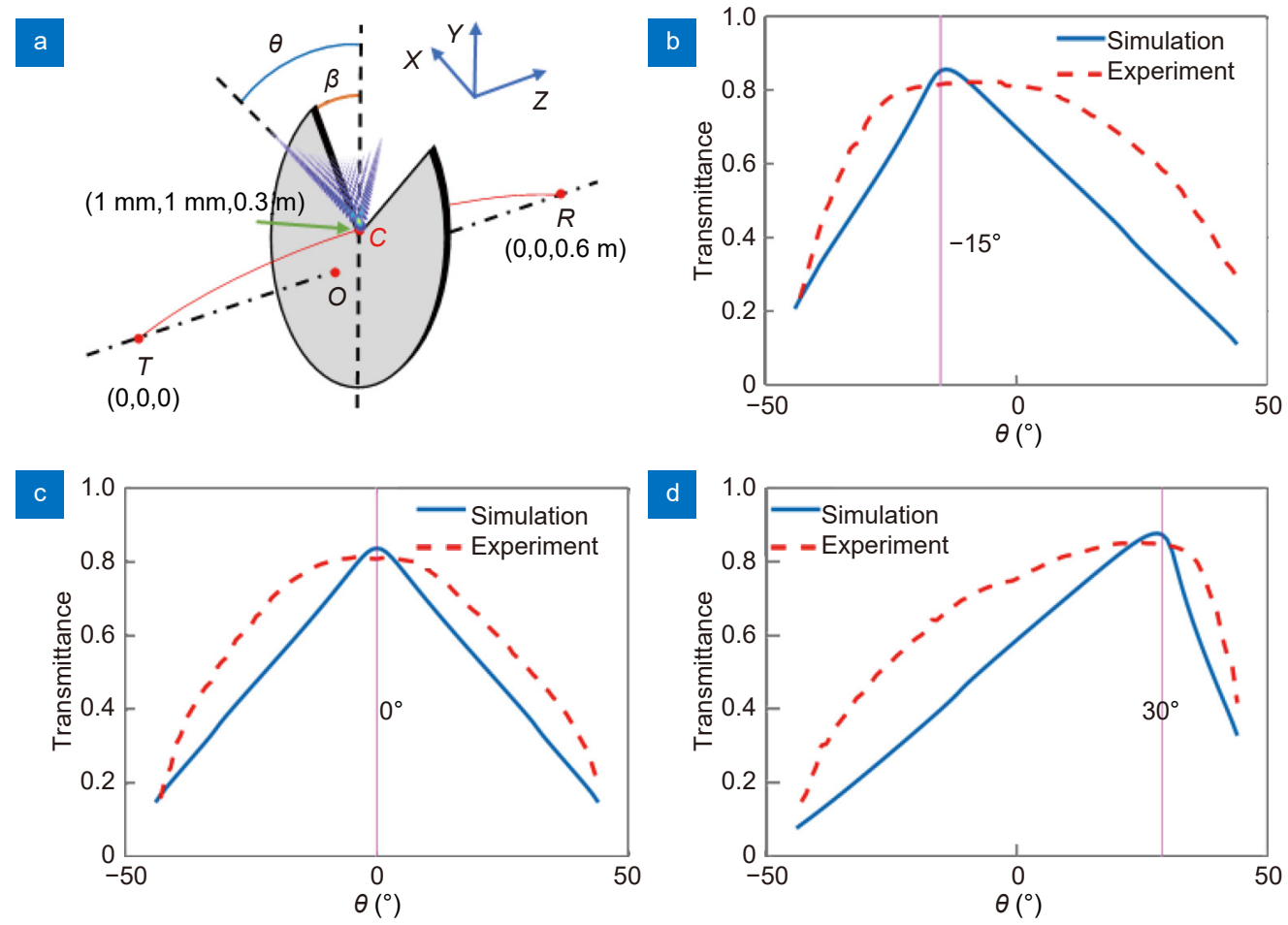

Fig. 5 | Obstacle evasion experiment. (a) set-up; normalized received optical power with obstacle's angle $\beta$ of (b) $-15^{\circ}$ (c) $0^{\circ}$ and (d) $30^{\circ}$. (blue solid curves are calculated results, and red dotted curves denote the experimental results.) 
self-accelerating beam with a single convex trajectory, we verify the scheme numerically and experimentally. Since the operation principle of the proposed method is based on the caustic analysis, it can apply to other self-accelerating beams. The proposed scheme to flexibly manipulate the rotation of transverse optical field is helpful for some applications sensitive to the optical field distribution for $2 \mathrm{D}$ self-accelerating beams, such as optical tweezers and laser micro-machining.

\section{References}

1. Bandres MA, Kaminer I, Mills M, Rodríguez-Lara BM, Greenfield $\mathrm{E}$ et al. Accelerating optical beams. Opt Photonics News 24, 30-37 (2013).

2. Rosen J, Yariv A. Snake beam: a paraxial arbitrary focal line. Opt Lett 20, 2042-2044 (1995).

3. Siviloglou GA, Broky J, Dogariu A, Christodoulides DN. Observation of accelerating Airy beams. Phys Rev Lett 99, 213901 (2007).

4. Siviloglou GA, Christodoulides DN. Accelerating finite energy Airy beams. Opt Lett 32, 979-981 (2007).

5. Jia S, Vaughan JC, Zhuang XW. Isotropic three-dimensional super-resolution imaging with a self-bending point spread function. Nat Photonics 8, 302-306 (2014).

6. Zhao JY, Chremmos ID, Song DH, Christodoulides DN, Efremidis NK et al. Curved singular beams for three-dimensional particle manipulation. Sci Rep 5, 12086 (2015).

7. Abdollahpour D, Suntsov S, Papazoglou DG, Tzortzakis S. Spatiotemporal airy light bullets in the linear and nonlinear regimes. Phys Rev Lett 105, 253901 (2010).

8. Chong A, Renninger WH, Christodoulides DN, Wise FW. AiryBessel wave packets as versatile linear light bullets. Nat Photonics 4, 103-106 (2010).

9. Mathis A, Courvoisier F, Froehly L, Furfaro L, Jacquot M et al. Micromachining along a curve: Femtosecond laser micromachining of curved profiles in diamond and silicon using accelerating beams. Appl Phys Lett 101, 071110 (2012)

10. Zhu GX, Wen YH, Wu X, Chen YJ, Liu L et al. Obstacle evasion in free-space optical communications utilizing Airy beams. Opt Lett 43, 1203-1206 (2018).

11. Liang $Y, H u Y$, Song $D H$, Lou $C B$, Zhang $X Z$ et al. Image signal transmission with Airy beams. Opt Lett 40, 5686-5689 (2015).

12. Bar-Ziv U, Postan A, Segev M. Observation of shape-preserving accelerating underwater acoustic beams. Phys Rev $B$
92, 100301 (2015).

13. Voloch-Bloch N, Lereah Y, Lilach Y, Gover A, Arie A. Generation of electron Airy beams. Nature 494, 331-335 (2013).

14. Salandrino A, Christodoulides D. Airy plasmons defeat diffraction on the surface. Physics 4, 69 (2011).

15. Zheng $Y, W u ~ Z F$, Shum PP, Xu ZL, Keiser G et al. Sensing and lasing applications of whispering gallery mode microresonators. Opto-Electron Adv 1, 180015 (2018).

16. Salandrino A, Christodoulides DN. Airy plasmon: a nondiffracting surface wave. Opt Lett 35, 2082-2084 (2010).

17. Kaminer I, Bekenstein R, Nemirovsky J, Segev M. Nondiffracting accelerating wave packets of Maxwell's equations. Phys Rev Lett 108, 163901 (2012).

18. Zhang $\mathrm{P}, \mathrm{Hu}$ Y, Li TC, Cannan D, Yin XB et al. Nonparaxial Mathieu and weber accelerating beams. Phys Rev Lett 109, 193901 (2012).

19. Bandres MA, Rodríguez-Lara BM. Nondiffracting accelerating waves: weber waves and parabolic momentum. New J Phys 15, 013054 (2013).

20. Berry MV, Upstill C. IV Catastrophe optics: morphologies of caustics and their diffraction patterns. Prog Opt 18, 257-346 (1980).

21. Vaveliuk P, Lencina A, Rodrigo JA, Matos OM. Caustics, catastrophes, and symmetries in curved beams. Phys Rev A 92, 033850 (2015).

22. Greenfield E, Segev M, Walasik W, Raz O. Accelerating light beams along arbitrary convex trajectories. Phys Rev Lett 106, 213902 (2011).

23. Melamed T, Shlivinski A. Practical algorithm for custom-made caustic beams. Opt Lett 42, 2499-2502 (2017).

24. Wen YH, Chen YJ, Zhang YF, Chen H, Yu SY. Winding light beams along elliptical helical trajectories. Phys Rev A 94, 013829 (2016).

25. Guillemin V, Sternberg S. Geometric Asymptotics (American Mathematical Society, Providence, 1977).

26. Wen YH, Chen YJ, Zhang YF, Chen H, Yu SY. Tailoring accelerating beams in phase space. Phys Rev A 95, 023825 (2017).

27. Bastiaans MJ. Application of the Wigner distribution function to partially coherent light. J Opt Soc Am A 3, 1227-1238 (2008).

\section{Acknowledgements}

We are grateful for financial supports from National Key R\&D Program of China (Grant No. 2018YFB1801001), the National Natural Science Foundation of China (Grant No. 61875061), and the Program for Guangdong Introducing Innovative and Entrepreneurial Teams.

\section{Competing interests}

The authors declare no competing financial interests. 